\title{
Prediction of miRNA in HIV-1 genome and its targets through artificial neural network: a bioinformatics approach
}

\author{
Manish Kumar Gupta • Kavita Agarwal • \\ Nutan Prakash $\cdot$ Dev Bukhsh Singh • \\ Krishna Misra
}

Received: 11 June 2012/Revised: 12 August 2012 / Accepted: 17 August 2012

(C) Springer-Verlag 2012

\begin{abstract}
MicroRNAs (miRNA) are a class of non-coding RNA which inhibits the expression of a particular gene by the process of nucleotide-sequence-specific post-transcriptional gene silencing method. miRNAs are $\sim 21 \mathrm{nt}$ long non-coding RNAs that are derived from larger hairpin RNA precursors. The short length of the miRNA sequences and relatively low conservation of pre-miRNA sequences restrict the conventional sequence-alignment-based methods of finding only relatively close homologs. On the other hand, it has been reported that miRNA genes are more conserved in the secondary structure of their precursor rather than in primary sequences. Therefore, secondary
\end{abstract}

Electronic supplementary material The online version of this article (doi:10.1007/s13721-012-0017-3) contains supplementary material, which is available to authorized users.

M. K. Gupta $(\square)$

Department of Bioinformatics, University Institute of Engineering and Technology, Chhatrapati Shahu Ji Maharaj University, Kanpur, UP 208024, India

e-mail: manish.kumar.gupta@gmail.com

K. Agarwal

National Institute of Immunology, New Delhi 110067, India

N. Prakash

Department of Biotechnology, Shree M. \& N. Virani

Science College, Rajkot, Gujarat 360005, India

D. B. Singh

Department of Biotechnology, Institute of Biosciences

and Biotechnology, Chhatrapati Shahu

Ji Maharaj University, Kanpur, UP 208024, India

K. Misra

Centre of Biomedical Magnetic Resonance,

Sanjay Gandhi Post-Graduate Institute of Medical Sciences,

Campus Raebareli Road, Lucknow, UP 226014, India structural features should be fully exploited in the homologue search for new miRNA genes. In this study, an approach for identification and prediction of miRNA in viruses through artificial neural networks (ANN) has been proposed. This idea uses both sequential and structural features of pre-miRNA to train the ANN for identification of miRNA in new viral genomes. The designed ANN was found with an accuracy of $93.68 \%$ for the training dataset and $55.55 \%$ for the validation dataset. In case of HIV, this trained ANN identifies pre-miRNA which does not show sufficient homology to known pre-miRNA sequences, but are highly conserved in their structure. Finally, single miRNA of length 19 mer has been predicted targeting four genes namely NDUFS7, WNT3A, SUFU, and FOXK1 a strict threshold at score 19 . The results indicate that this method can be used for identifying novel miRNAs in other viral genomes with considerable success.

Keywords MicroRNA - Pre-miRNA - ANN · HIV · RNAFold · MiRBASE - Non-coding RNA · Gene target

\section{Introduction}

MicroRNAs (miRNA) are small regulatory RNAs, $\sim 21$ nucleotides in length, processed from short stem-loop precursors that are encoded in genomes of metazoans and viruses. The genes of miRNA transcribe into primary miRNA which process to form pre-miRNA, having length $\sim 70$ with stem loop structure with help of RNaseIII enzyme Drsoha in the animal cells. Later, Dicer which is a class of RNaseIII enzyme (Grishok et al. 2001; Hutvagner et al. 2001; Ketting et al. 2001) incises the pre-miRNAs to release the $\sim 21$ nucleotides mature miRNAs (Lee et al. 2002, 2003). At the end, RNA-induced silencing complexes 
(RISC) (Hammond et al. 2000) are produced to regulate the expression of target genes via complementary base pair interactions. The precursor lengths of miRNA are more variable and their structures are more complex in plants, but the maturation process of miRNA is comparable with that of Animals. miRNAs can bind to the $3^{\prime}$ UTRs of messenger RNAs (mRNAs) and interfere with their translation, thus contributing a significant post-transcriptional regulatory step in gene expression. They have been shown to play an important role at the level of development, apoptosis, and establishment of cell lineage in various organisms (He and Hannon 2004; Bartel 2004). Human immunodeficiency virus-1 (HIV-1) infects human cells and incorporates its DNA into the host genome. HIV-1 infection stimulates expression of particular sets of cellular miRNAs, including miR-29a. HIV-1 mRNA is transcribed, exported from the nucleus, and translated into viral proteins. Cellular RISC having specific miRNAs, such as miR-29a, target HIV-1 mRNA and sequestering the ribonucleoprotein (RNP) complex in $\mathrm{P}$ bodies. Depending upon cellular stimuli or viral pathogenesis signs, HIV-1 mRNA could be stored in $\mathrm{P}$ bodies and released for subsequent translation of viral proteins. Alternatively, viral mRNA could be degraded in $P$ bodies (Nathans et al. 2009).

Although the exact mechanism by which miRNA mediates regulation is not completely understood, several experimental observations have been made which generalize the rules of miRNA-transcript binding. Most important among them are the incomplete complementarity of pairing and the initial continuous base pairing, referred to as the seed with base $2-8$ of the $5^{\prime}$ end of the miRNA pairing completely with the $3^{\prime}$ UTR of the transcript. Other general features like optimum minimum free energy (MFE) of the bound complex and conservation in related sequences also favor miRNA function. HIV-1 has been identified as an etiological agent responsible for acquired immune deficiency syndrome (AIDS), a fatal condition that arises by the invasion of the virus on various cells of the human immune system (Arendt and Littman 2001; Emerman and Malim 1998). It has been reported that only copious miRNA genes can be easily detected by Polymerase Chain Reaction or northern blot due to limitations of the techniques. For finding those low-expression or tissue-specific miRNA genes computational prediction provides an efficient strategy (Bartel 2004). Several miRNAs have been accounted in the herpesvirus family (Pfeffer et al. 2005). A computational approach has already been developed for predicting miRNA (Lim et al. 2003a, b; Lai et al. 2003; Thomassen et al. 2006) in animals considering both sequence and structure alignment (Wang et al. 2005).

Artificial neural networks are a form of artificial intelligence that can learn to predict, through modeling, answers to particular questions in complex data. The models produced by ANNs have been shown to have the ability to predict well for unseen data and have the ability to cope with complexity and nonlinearity within the dataset; these features of ANNs mean they have the potential to identify and model patterns in this type of data to address a particular question (Anderson and McNeill 1995; Khan et al. 2001). In this study, we have developed an approach for computational identification of miRNA, which utilizes the knowledge from homology-based search methods and machine learning approaches along with knowledge-based feature selection methods (Van Hulse et al. 2012; Liou and Huang 2012; Zillner and Sonntag 2012).

A significant number of miRNAs have been reported experimentally but majority of gene target are still unknown. So, Insilico target prediction tools remain the only way for a rapid identification of miRNA target (Kiriakidou et al. 2004, Alexiou et al. 2009).

\section{Materials and methods}

\subsection{Data collection}

The nucleotide sequences of pre-miRNAs, mature miRNA and annotated information were retrieved from miRBase database (http://www.microrna.sanger.ac.uk/sequences), which contains all experimentally verified data from 11 different viral genomes (Griffiths-Jones et al. 2006, 2008; Ambros et al. 2003; Kozomara and Griffiths-Jones 2011; Griffiths-Jones 2004). Total 109 pre-miRNA sequences were collected from different viral genomes given in Table 1.

\subsection{Secondary structure extraction}

The RNA secondary structure includes external elements (non-paired bases), double strand or stacks (paired bases), a hairpin (a double strand and a loop), bulge loops, interior loops, and multi-loops. RNA can also have tertiary interaction, such as pseudoknots. The RNAfold program (Gruber et al. 2008) has been used for the extraction RNA secondary structure on the Linux operating system. The structure-based features were extracted by programs written in Perl programming language. The input to the RNAfold program was FASTA format of precursor sequences.

\subsection{Classifier}

The ANN classifier has been used in this computational approach which is written in Perl programming language. A three-layer ANN architecture (Multi-Layer Perceptron) (Anderson and McNeill 1995) has been adopted for the analysis of secondary structure. The input layer consists of 
Table 1 Name of virus family and their miRNAs

\begin{tabular}{|c|c|c|c|c|}
\hline S. no & Virus family & Species & Number & miRNAs \\
\hline \multirow[t]{10}{*}{1.} & \multirow[t]{10}{*}{ Herpesvirus } & Epstein-barr virus (EBV) & 23 & miR-BHRF1-(1-3) \\
\hline & & & & miR-BART(1-20) \\
\hline & & $\begin{array}{l}\text { Kaposi-sarcoma associated } \\
\text { herpes virus (KSHV) }\end{array}$ & 13 & miR-K12(1-9,10a,10b,11-12) \\
\hline & & Human cytomegalovirus (HCMV) & 11 & $\begin{array}{l}\text { miR-UL22A, miR-US5-1, } \\
\text { miR-US5-2, miR-UL36, } \\
\text { miR-UL112, miR-US25-1, } \\
\text { miR-US25-2, miR-UL148D, } \\
\text { miR-US33, miR-UL70, } \\
\text { miR-US4 }\end{array}$ \\
\hline & & Mareks disease virus (MDV1) & 8 & Mdv1-miR-M(1-8) \\
\hline & & Mareks disease virus type 2 (MDV2) & 17 & Mdv2-miR-M(14-30) \\
\hline & & Herpes simplex virus 1 (HSV-1) & 2 & miR-LAT, miR-H1 \\
\hline & & $\begin{array}{l}\text { Mouse gamma herpes virus } 68 \\
\text { (MGHV68) }\end{array}$ & 9 & miR-M1-(1-9) \\
\hline & & Rhesus lymphocryptovirus (RLCV) & 16 & miR-rL1-(1-16) \\
\hline & & Rhesus monkey rhadinovirus (RRV) & 7 & miR-rR1-(1-7) \\
\hline 2. & Polyomavirus & Simian virus 40 (SV40) & 1 & miR-S1 \\
\hline 3. & HIV & HIV-1 & 2 & miR-N367, miR-H1 \\
\hline
\end{tabular}

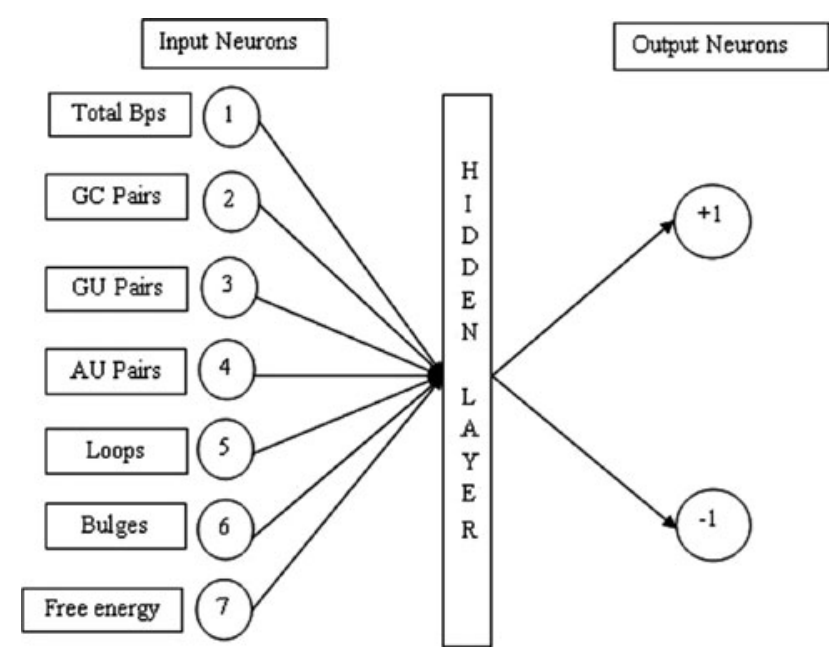

Fig. 1 Architecture of artificial neural network and input parameter

seven neurons for each pattern, corresponding to the seven secondary structure features of pre-miRNA (Fig. 1). The output layer consists of single neuron indicating whether a potential pre-miRNA candidate can actually act as a premiRNA. A value of plus one indicates that the sequence can act as a pre-miRNA while minus one means that the sequence cannot act as a pre-miRNA.

\subsection{Pre-miRNA prediction}

It is important to predict Pre-miRNA before mature miRNA. A rigorous method has been applied for identification of Pre-miRNA. The existing Pre miRNA structures were taken for extracting features such as GC, GU, AU pairs, loops, bulges, total base pair, and free energy. Data set related to each structural feature was normalized to get optimum value. The normalized dataset was segregated into training and validation dataset. Back-propagation algorithm was applied to train the training dataset. Finally, the output of trained network was correlated with potential precursor. The Computational steps taken into account for prediction of pre- miRNA in HIV-1 genome are shown in Fig. 2.

\section{5 miRNA target prediction}

The DIANA-microT v 3.0 web server (http://diana.cslab. ece.ntua.gr/microT/microT.php) was used to predict possible target genes of differentially expressed miRNAs. DIANA-microT 3.0 prediction algorithm is primarily based on dynamic programming routines. The prediction score of a miTG interaction is the weighted sum of the scores of conserved and non-conserved miRNA-recognition elements (MRE) on a gene. Diana-microT identifies miRNA targets by combining TargetScan and PicTar predictions and has been reported to be the most selective algorithm in a comparative study (Maragkakis et al. 2009a, b). The predicted miRNA sequence is subjected for target gene target identification in FASTA format to DIANA-microT server. 


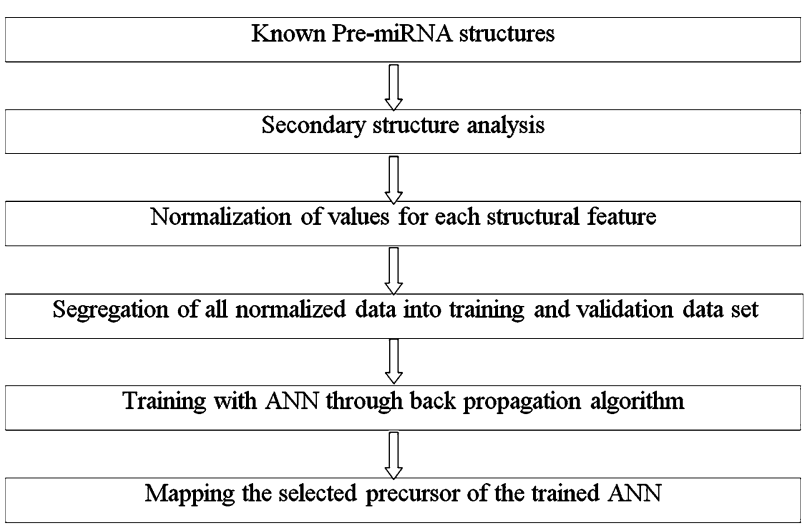

Fig. 2 Methodology for training the neural network

\section{Results and discussion}

\subsection{Known pre-miRNA feature extraction}

The sequential data of both pre-miRNA and mature miRNA were analyzed with local programs written using Perl programming language. The analysis showed that the average length of the known pre-miRNA sequences is 78 nucleotides and average GC content in any pre-miRNA sequence is $42 \%$. The stem-loop structures of pre-miRNAs were analyzed for the total number of base pairing in the stem region, GC pairs in the stem region, GU pairs in the stem region, Total number of Loops in the structure, Total number of Bulges in the structure, and Free Energy of the secondary structure. The free energy of the RNA secondary structure varies from -21.70 to $-59.20 \mathrm{kcal} /$ $\mathrm{mol}$ and average energy value is found $-36.58 \mathrm{kcal} / \mathrm{mol}$ (Fig. 3).

The values obtained for each parameter have been represented in the form of histogram and analyzed statistically. The quantitative analysis indicates that average number of base pairing in the stem region is 28 with a standard deviation of 4.6580. The Maximum and minimum number of base pair in the stem region were found 43 and 18, respectively (Fig. 4). The other parameters like GC, GU, AC pairs loop, and bulges were also estimated.

The length of precursor varies from 57 (hsv1-mir-LAT MI0006105) to 120 (mdv1-mir-M6 MI0005098). The maximum base pair of precursor miRNA existing in EBV (Accession no. MI0004991) is 43. The Standard deviation value of GU pairing is 1.7473 which can be further minimized by considering more number of experimentally identified miRNA in virus (Supplementary Table 1).

\subsection{Data preparation}

Information based on existing precursor miRNA of the viral genomes in miRBase, two dataset was prepared, one

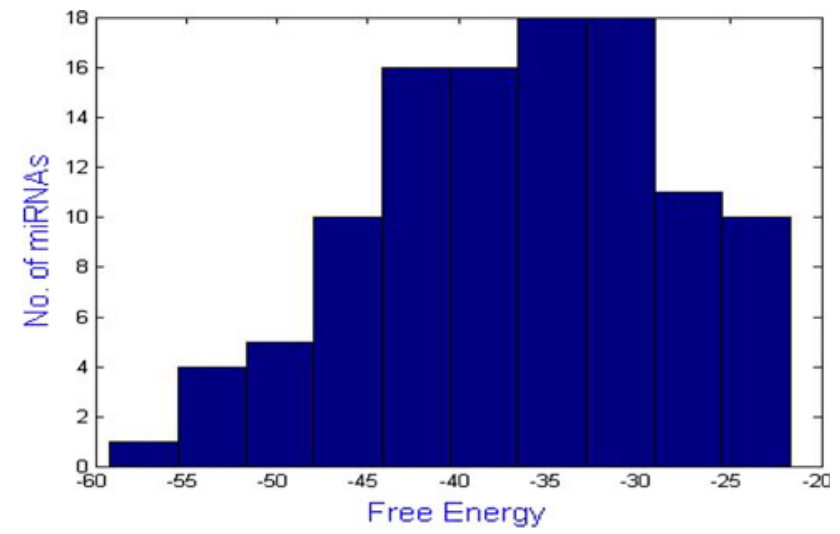

Fig. 3 Histogram of free energy $(\mathrm{kcal} / \mathrm{mol})$ of the stem-loop structure of the training set of pre-miRNA

for training and one for validation. Both the data sets contain mutually exclusive information. In the training dataset normalized values, seven structural features, were used so that the varying number of pre-miRNA from each viral genome did not over train the network for a particular viral species. The network was trained on 190 patterns, among which 100 belonged to known pre-miRNA sequences (having a target output neuron value of 1) and 90 patterns were based on the sequences that were identified as non pre-miRNA sequences in the viral genome (having a target output neuron value of -1 ) (Table 2).

\subsection{Training of ANN}

The back-propagation algorithm was used to train the neural network. The parameters used for training are given in the Table 3 . The training process starts by assigning arbitrary initial connection weights, which are then updated until an acceptable training accuracy is achieved. Overall root mean square error (RMS) was calculated after each epoch, and the weights were updated accordingly, both, between the hidden-output nodes as well as between the input-hidden nodes. After training the network once through all the input node (an epoch) the RMS is calculated for all the input nodes. The ANN was trained for 200 epochs in the first attempt but on increasing the number of epochs to 500 the RMS decreases further. The training data reveal that the RMS is very low $(<0.0019)$ (Table 4). Once the training process is done, the generalization in the network has been evaluated by running the test dataset through the network. The trained ANN performs well on the training data as well as on validation data. Overall, an accuracy of $93.68 \%$ was achieved in predicting the sequences of training set and an accuracy of $55.55 \%$ on the validation dataset (Table 5).

The neural networks developed during this effort were generated from data found mainly in the online databases. 
Fig. 4 Total base pairing in the stem region in precursor miRNA

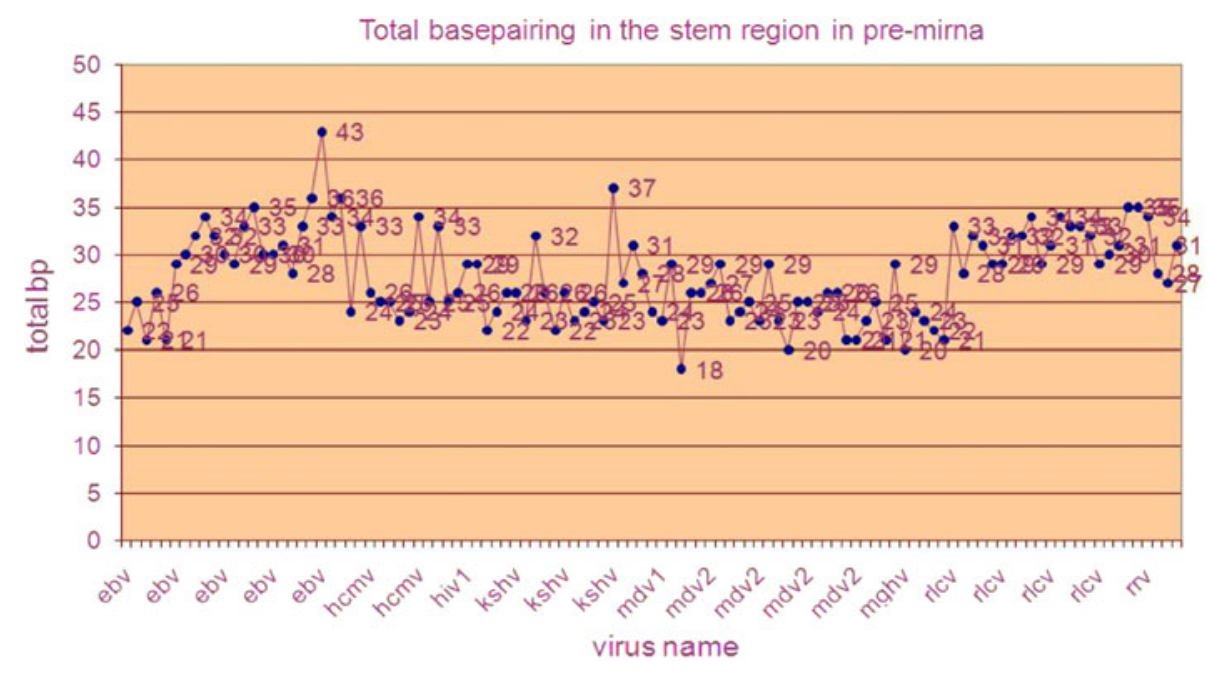

Table 2 Input parameter for positive training data set

\begin{tabular}{|c|c|c|c|c|c|c|c|c|c|c|}
\hline S. no & Virus name & Accession no. & Total bps & GC pairs & GU pairs & AU pairs & Loops & Bulges & Free energy & Output \\
\hline 1. & ebv & 1064 & -1.23099 & -1.6104 & -0.72981 & 0.475907 & -0.82619 & -0.2609 & 1.336137 & 1 \\
\hline 2. & ebv & 1065 & -0.58693 & -2.15724 & 0.987087 & 1.140956 & -0.82619 & -1.49731 & 1.143249 & 1 \\
\hline 3. & ebv & 1066 & -1.44567 & -1.33699 & -0.72981 & -0.18914 & 0.498146 & -1.49731 & 1.613414 & 1 \\
\hline 4. & ebv & 1067 & -0.37225 & -0.24332 & -0.15751 & -0.18914 & 0.498146 & 0.357314 & 0.68514 & 1 \\
\hline 5. & ebv & 1068 & -1.44567 & -1.6104 & -1.30211 & 0.475907 & -0.82619 & -0.87911 & 1.287915 & 1 \\
\hline 6. & ebv & 3725 & 0.271802 & 0.030101 & 0.414786 & 0.143382 & -0.82619 & -0.2609 & -0.4963 & 1 \\
\hline 7. & ebv & 3726 & 0.486485 & -0.24332 & 0.987087 & 0.475907 & -0.82619 & 0.357314 & -0.2793 & 1 \\
\hline 8. & ebv & 3727 & 0.915853 & 0.850353 & 0.414786 & 0.143382 & 0.498146 & 0.975523 & -0.24313 & 1 \\
\hline 9. & ebv & 3728 & 1.345221 & -0.24332 & 0.414786 & 2.13853 & 1.822485 & -0.87911 & -0.9303 & 1 \\
\hline 10. & ebv & 3729 & 0.915853 & 0.303518 & -1.30211 & 1.806005 & -0.82619 & 1.593733 & -0.42397 & 1 \\
\hline 11. & ebv & 3730 & 0.486485 & -1.06301 & 1.568966 & 1.146667 & -0.82667 & 0.360248 & 0.564536 & 1 \\
\hline 12. & ebv & 3731 & 0.27897 & 0.032877 & -1.3046 & 1.146667 & 1.84 & -0.26087 & 0.323281 & 1 \\
\hline 13. & ebv & 3732 & 1.137339 & 0.580822 & -0.15517 & 1.146667 & -0.82667 & 0.981366 & -1.60676 & 1 \\
\hline 14. & ebv & 3733 & 1.566524 & 1.128767 & 2.143678 & -0.18667 & 0.506667 & 0.360248 & -0.83474 & 1 \\
\hline 15. & ebv & 3734 & 0.493562 & 0.580822 & 0.41954 & -0.18667 & 0.506667 & 0.360248 & -0.2316 & 1 \\
\hline 16. & ebv & 3735 & 0.493562 & -0.2411 & 0.994253 & 0.48 & 0.506667 & 1.602484 & 0.648975 & 1 \\
\hline 17. & ebv & 3736 & 0.708155 & 0.854795 & -0.15517 & 0.146667 & -0.82667 & 1.602484 & 0.045838 & 1 \\
\hline 18. & ebv & 4988 & 0.064378 & -0.51507 & -0.15517 & 0.813333 & 0.506667 & 0.360248 & 0.902292 & 1 \\
\hline 19. & ebv & 4989 & 1.137339 & 0.306849 & -1.3046 & 2.146667 & 1.84 & 0.360248 & 0.214717 & 1 \\
\hline 20. & ebv & 4990 & 1.781116 & 1.128767 & 0.41954 & 1.146667 & 1.84 & -0.26087 & -1.57057 & 1 \\
\hline 21. & ebv & 4991 & 3.283262 & 1.128767 & 3.293103 & 1.813333 & 0.506667 & 1.602484 & -1.23281 & 1 \\
\hline 22. & ebv & 4992 & 1.351931 & 0.032877 & 0.994253 & 1.48 & 0.506667 & 0.360248 & -0.64174 & 1 \\
\hline 23. & ebv & 4993 & 1.781116 & 1.40274 & -0.72989 & 1.48 & 1.84 & 0.981366 & -0.60555 & 1 \\
\hline
\end{tabular}

For refined algorithm development, experimentally verified data are always preferred even though the accuracy of a database is high. The candidate sequences for which the neural model output was very close to 1(i.e. greater than 0.98 ) were taken to be pre-miRNA sequences. All the other sequences were rejected as they either showed neural model output which is approximately to -1 or very low positive values (Fig. 5).
The positive data train the neural network to identify true positives while the negative training set helps in identification of true negatives and decreases the chances of getting a false positive. Overall, the negative training data set is an essential part to improve the specificity of the program. In addition, the negative training data used for training and evaluating the ANN are almost equal in proportion to the positive training data. In biological systems, 
Table 3 Parameters and Values used to train the ANN

\begin{tabular}{lll}
\hline S. no & Training parameters & Value used \\
\hline 1. & numEpochs & 500 \\
2. & numInputs & 7 \\
3. & numPatterns & 190 \\
4. & Input-hidden $(\mu)$ & 0.1 \\
5. & Hidden-output $(\delta)$ & 0.01 \\
\hline
\end{tabular}

Table 4 Result of RMS error value for each epoch of Training data

\begin{tabular}{|c|c|c|}
\hline S. no. & Epoch & RMS error \\
\hline 1. & 159 & 0.000654505722655954 \\
\hline 2. & 160 & 0.00148536777823122 \\
\hline 3. & 161 & 0.00129096123728604 \\
\hline 4. & 162 & 0.000716927807938541 \\
\hline 5. & 163 & 0.00057824377371156 \\
\hline 6. & 164 & 0.000867408564513525 \\
\hline 7. & 165 & 0.00220175381918606 \\
\hline 8. & 166 & 0.00302090104138388 \\
\hline 9. & 167 & 0.00126386479550477 \\
\hline 10. & 168 & 0.00145948386600876 \\
\hline 11. & 169 & 0.00103814083153027 \\
\hline 12. & 170 & 0.00299727776471991 \\
\hline 13. & 171 & 0.000559504458559562 \\
\hline 14. & 172 & 0.00229944213505394 \\
\hline 15. & 173 & 0.017734715360151 \\
\hline 16. & 174 & 0.000951223162028956 \\
\hline 17. & 175 & 0.00259875067870514 \\
\hline 18. & 176 & 0.000914788006246549 \\
\hline 19. & 177 & 0.00106742605422625 \\
\hline 20. & 178 & 0.00108476283232372 \\
\hline 21. & 179 & 0.0191880541210997 \\
\hline 22. & 180 & 0.00836210042034909 \\
\hline 23. & 181 & 0.0073024535984521 \\
\hline
\end{tabular}

Table 5 Results obtained on training the ANN

\begin{tabular}{lllll}
\hline S. no. & Datasets & $\begin{array}{l}\text { Correct } \\
\text { predictions }\end{array}$ & $\begin{array}{l}\text { Incorrect } \\
\text { predictions }\end{array}$ & $\begin{array}{l}\text { Accuracy } \\
(\%)\end{array}$ \\
\hline 1. & Training & 178 & 12 & 93.68 \\
2. & Validation & 5 & 4 & 55.55 \\
\hline
\end{tabular}

pre-miRNA coding regions are much less in number as compared with non-coding regions. The effect of these regions on the identification of pre-miRNA coding regions can be evaluated by increasing the proportion of negative training data. An exciting advancement would be pattern recognition of these elements in higher organisms. premiRNA by itself does not show much homology in sequence, or one can say that mature miRNA sequences are embedded in very divergent kind of pre-miRNAs. Although the training results of the neural network show low RMS error (Table 4) still the training can be improved by adding more data in the negative training set from other Viral genomes.

\subsection{Analysis of pre-miRNA}

The pre-miRNA nucleotide sequences of 109 viruses were used as nucleotide query to BLAST (Altschul et al. 1990) against the HIV-1 complete genome (gil4558520lgblAF033819.3I). A total of 528 homologues were taken for further analysis, with $E$ value less than or equal to 2.8; others with much higher $E$ values were discarded (Supplementary Table 2). $E$ value explains the random background noise. $E$-value having lower or closer to zero is more significant to the match. On including the sequences with higher $E$ values the redundancy increased in the data as many sequences were similar. 285 homologues were identified on the plus strand and 243 homologues on the minus strand of the HIV genome. The homologues obtained in the BLAST output were small sequences of 10-15 nucleotides in length; therefore, potential pre-miRNA sequences were extracted from the genome by flanking 30 nucleotides on each side of the homologue found on both the strands. The homologs that were identified in the HIV genomes against BLAST were found very short in their length which indicates that pre-miRNA by itself does not show much homology in sequence or one can say that mature miRNA sequences are embedded in very divergent kind of pre-miRNAs. Homology-based methods are helpful to scan newly sequenced genomes for homologues of already existing miRNA and alternatively find the miRNA gene in earlier studied genomes (Mendes et al. 2009).

After the secondary structure prediction, the potential pre-miRNA candidates were filtered based on their free energy. All the secondary structures with estimated free energy value less than or equal to $-20.90 \mathrm{kcal} / \mathrm{mol}$ were taken out for further study. A total of 25 candidates (Table 7) were filtered from precursors obtained on the minus strand and 13 candidates (Table 6) were obtained from precursors obtained on the plus strand. Therefore, this step helped greatly in reducing the search space from 528 homologues obtained as output of the BLASTn program to 38 potential pre-miRNA candidates.

The potential pre-miRNA candidates that were obtained in the previous step were mapped to the neural network with their structural features. Therefore, from 38 potential candidates only two sequences were predicted to pre-miRNA on mapping to the ANN. On mapping these pre-miRNA structures to the trained ANN, two pre-miRNAs were identified as the best candidate for miRNA. The 
Table 613 Potential pre-miRNA with estimated free energy values of secondary structure on plus strand

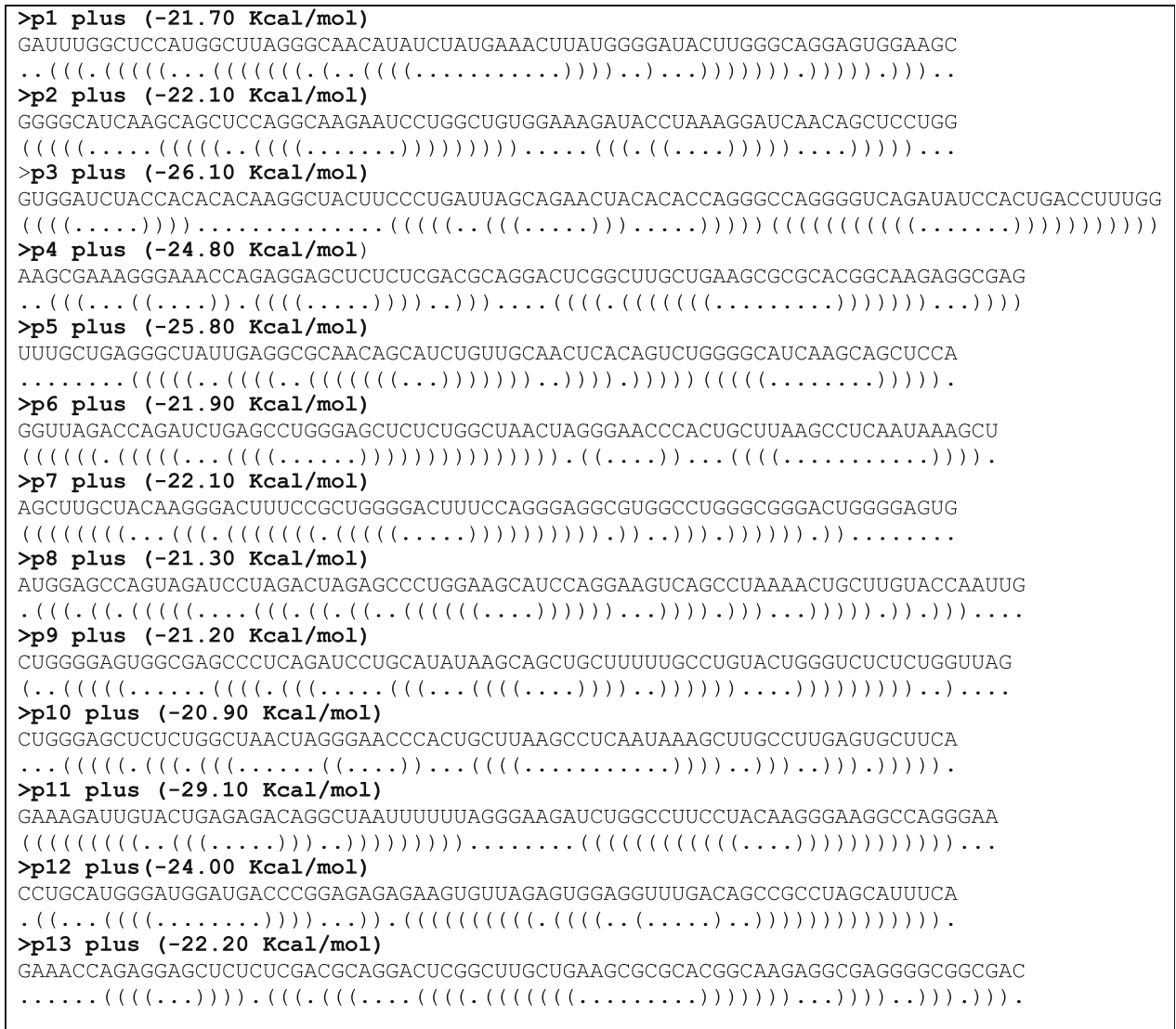

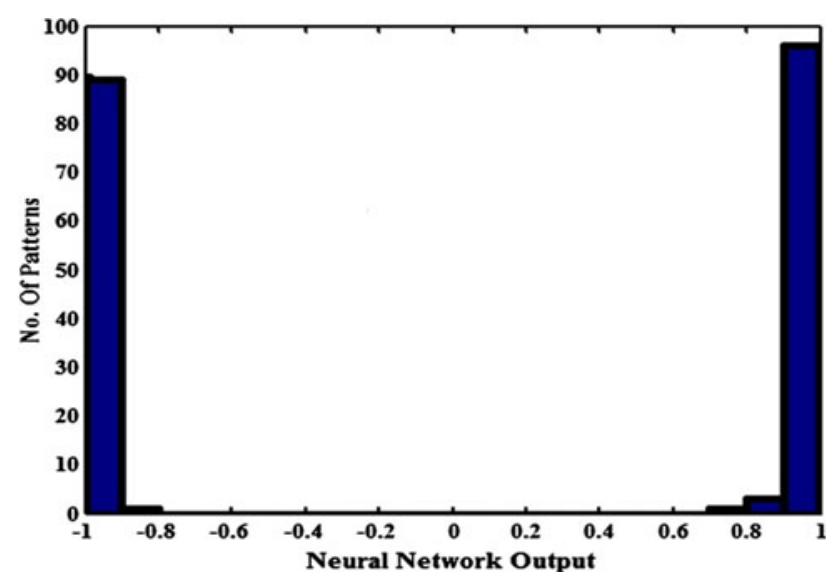

Fig. 5 Output of ANN on the training dataset

pre-miRNA predicted on the plus strand of the HIV genome is having free energy of $-22.10 \mathrm{kcal} / \mathrm{mol}$. The pre-miRNA predicted on the minus strand of the HIV genome is having free energy of $-22.40 \mathrm{kcal} / \mathrm{mol}$.

$$
>\text { p7 plus }(-22.10 \mathrm{kcal} / \mathrm{mol})
$$

AGCUUGCUACAAGGGACUUUCCGCUGGGGACUU UCCAGGGAGGCGUGGCCUGGGCGGGACUGGGGA GUG

\section{$>$ p15 minus $(-22.40 \mathrm{kcal} / \mathrm{mol})$ ACCUUCUUCUUCUAUUCCUUCGGGCCUGUCGGG UCCCCUCGGGGUUGGGAGGUGGGUCUGAAACG AUAA}

Besides p7 and p15 pre-miRNA, p11 $(-29.10 \mathrm{kcal} / \mathrm{mol})$ from plus stand and p17 $(-26.80 \mathrm{kcal} / \mathrm{mol})$ from minus strand were also considered because of lowest energy. The predicted pre-miRNA from ANN, p11 from plus strand and p17 from minus strand were aligned with miRBase database using BLASTN program. After alignment of $\mathrm{p} 7$ of plus strand with mature miRNA of miRBase database, the $E$ value was found to be 0.92 . The significant $E$ value (1e-05) was found when $\mathrm{p} 7$ aligned with stem loop sequence of miRbase database. The $E$ value for $\mathrm{p} 7 \mathrm{stem}$ loop sequence is lowest among all the alignment of p11 (+strand), p15 (-strand), and p17 (-strand). The alignment score of $\mathrm{p} 7(+)$ aligned with miRbase is also very high among all the alignments made (Table 8 ). This suggests that the mature predicted miRNA is embedded in the precursor sequence of $\mathrm{p} 7$.

The computational analysis indicates that the $\mathrm{p} 7$ have shown significant similarity with hiv1-miR-H1 miRNA of mirRBase database, which validates the presence of predicted miRNA in HIV-1 genome. The predicted miRNA 
Table 725 Potential pre-miRNA with estimated free energy values of secondary structure on minus strand

$>\mathrm{p} 1$ minus $(-24.00 \mathrm{Kcal} / \mathrm{mol})$

AAGAGGCACAGGCUCCGCAGAUCGUCCCAGAUAAGUGCCAAGGAUCCGUUCACUAAUCGAAUGGAUCUGUCU $\ldots(((((\ldots((\ldots)) \ldots(((\ldots \ldots))) \ldots))))) \ldots((((((((((\ldots \ldots)))))))))) \ldots$ $>\mathrm{p} 2$ minus $(-21.80 \mathrm{Kcal} / \mathrm{mol})$

UUUACAAUAGCAAUUGGUACAAGCAGUUUUAGGCUGACUUCCUGGAUGCUUCCAGGGCUCUAGUCUAGGAUCU $\ldots \ldots((((\ldots)))) \ldots \ldots \ldots(((((((((((\ldots)((((((\ldots \ldots)))))))) \ldots))))))))))) \ldots$ $>$ p3 minus $(-22.40 \mathrm{Kcal} / \mathrm{mol})$

CAACUGGUACUAGCUUGUAGCACCAUCCAAAGGUCAGUGGAUAUCUGACCCCUGGCCCUGGUGUGUAGUU $\left.\left.\left.\ldots \ldots \ldots \ldots\left(\left(\left((\ldots \ldots)\left(\left(\left(\left(\left({ }_{1}\left(\left(\left(\ldots\left(\left(\left(\left(\left(\ldots_{1} \ldots\right)\right)\right)\right)\right)\right) \ldots\right)\right)\right) \ldots\right)\right)\right)\right)\right)\right) \ldots\right)\right)\right)\right)$ $>$ p4 minus $(-23.30 \mathrm{Kcal} / \mathrm{mol})$

uCCUGCUUCUGAgGGGGAguUguUguCUCUACCCCAGACCUGAAGCUCUCUUCUGGUGGGGCUGUUGGCUCUGGU $((((.((\ldots \ldots))))).) \ldots \ldots \ldots \ldots \ldots((((((\ldots \ldots(((((\ldots \ldots \ldots)))))) \ldots))))))$.$) .$ $>$ p5 minus $(-22.80 \mathrm{Kcal} / \mathrm{mol})$

UUGGCUCUGGUCUGCUCUGAAGAAAAUUCCCUGGCCUUCCCUUGUAGGAAGGCCAGAUCUUCCCUAAAAAAUUA $\ldots(((\ldots \ldots \ldots))) \ldots(((((\ldots \ldots \ldots((((((((((\ldots \ldots)))))))))))))))).) \ldots \ldots$ $>$ p 6 minus $(-25.10 \mathrm{Kcal} / \mathrm{mol})$

GGCUUAAGCAGUGGGUUCCCUAGUUAGCCAGAGAGCUCCCAGGCUCAGAUCUGGUCUAACCAGAGAGACC .$\left.((\ldots .).) \ldots\left(\left(\left(\ldots\left(\left(\left(.\left(\left(\left(\left(\left(\left(\left(\left(\left(\left(\left(\left(\left(\left({ }^{\prime} \ldots\right)\right)\right)\right)\right) \ldots\right)\right)\right)\right)\right).\right)\right)\right)\right)\right).\right)\right).\right) \ldots\right)\right)\right)$ $>$ p7 minus $(-22.30 \mathrm{Kcal} / \mathrm{mol})$

GGCUUAAGCAGUGGGUUCCCUAGUUAGCCAGAGAGCUCCCAGGCUCAGAUCUGGUCUAACCAGAGAGAC

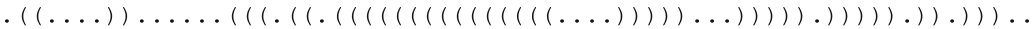
$>$ p8 minus $(-20.90 \mathrm{Kcal} / \mathrm{mol})$

GGCAAAAAGCAGCUGCUUAUAUGCAGGAUCUGAGGGCUCGCCACUCCCCAGUCCCGCCCAGGCCACGCC $(((\ldots((((\ldots)))) \ldots((.(((.(((.(((\ldots \ldots \ldots)))))))))).) \ldots \ldots))) \ldots \ldots$ $>$ p9 minus $(-21.20 \mathrm{Kcal} / \mathrm{mol})$

UCUUAUCUGGCUCAACUGGUACUAGCUUGUAGCACCAUCCAAAGGUCAGUGGAUAUCUGACCCCUGGCCCUGGUGU $\ldots \ldots((((\ldots(\ldots \ldots) \ldots)))) \ldots \ldots(((((\ldots(((\ldots(((((\ldots \ldots \ldots)))))) \ldots))) \ldots))))))$ $>$ p10 minus $(-20.90 \mathrm{Kcal} / \mathrm{mol})$

AAAAUUCCCUGGCCUUCCCUUGUAGGAAGGCCAGAUCUUCCCUAAAAAAUUAGCCUGUCUCUCAGUACAAU

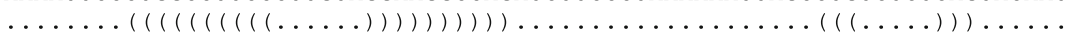
$>$ p11 minus $(-22.20 \mathrm{Kcal} / \mathrm{mol})$

ACAAGCAGUUUUAGGCUGACUUCCUGGAUGCUUCCAGGGCUCUAGUCUAGGAUCUACUGGCUCCAUUUCUU $\left.\ldots\left(\left(\left(.\left(\left(\left(\left(()\left(\left(\left(\left((\ldots)\left(\left(\left(\left(\left(\left({ }^{\prime} \ldots\right)\right)\right)\right)\right)\right)\right) \ldots\right)\right)\right)\right)\right)\right)\right)\right)\right)\right)\right) \ldots \ldots . \ldots$ $>$ p12 minus $(-23.00 \mathrm{Kcal} / \mathrm{mol})$

UUCUACUACUCCUUGACUUUGGGGAUUGUAGGGAAUUCCAAAUUCCUGCUUGAUUCCCGCCCACCAACAGGCGGCCCUAACC $\ldots \ldots \ldots \ldots \ldots \ldots(((((\ldots \ldots(((1((1 \ldots \ldots \ldots))))))) \ldots \ldots((((\ldots \ldots \ldots))))))))))) \ldots$ $>$ p13 minus $(-22.30 \mathrm{Kcal} / \mathrm{mol})$

UUGAGGCUUAAGCAGUGGGUUCCCUAGUUAGCCAGAGAGCUCCCAGGCUCAGAUCUGGUCUAACCAGAG $\ldots((((\ldots(((\ldots((\ldots)) \ldots))))))) \ldots((((\ldots))))) \ldots((((\ldots) \ldots)))))$. $>$ p14 minus $(-26.50 \mathrm{Kcal} / \mathrm{mol})$

CACCUUCUUCUUCUAUUCCUUCGGGCCUGUCGGGUCCCCUCGGGGUUGGGAGGUGGGUCUGAAACGAUAAUGGUGA

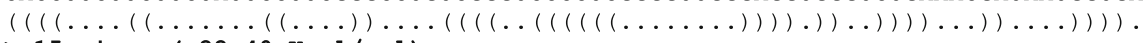
>p15 minus $(-22.40 \mathrm{Kcal} / \mathrm{mol})$

ACCUUCUUCUUCUAUUCCUUCGGGCCUGUCGGGUCCCCUCGGGGUUGGGAGGUGGGUCUGAAACGAUAA $((((\ldots(((((((\ldots)(((\ldots(((((\ldots \ldots))))) \ldots \ldots))) \ldots)))))))))).) \ldots \ldots$ $>$ p16 minus $(-23.20 \mathrm{Kcal} / \mathrm{mol})$

CUUCUUCUAUUCCUUCGGGCCUGUCGGGUCCCCUCGGGGUUGGGAGGUGGGUCUGAAACGAUAAUGGUGAAU

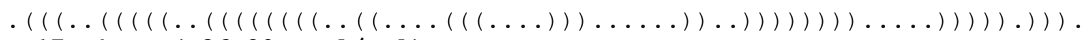
$>$ p17 minus $(-26.80 \mathrm{Kcal} / \mathrm{mol})$

ACAGUUCCUUGUCUAUCGGCUCCUGCUUCUGAGGGGGAGUUGUUGUCUCUACCCCAGACCUGAAGCUCUCUUCUGGUGGGG $(((\ldots(((((.((\ldots \ldots(((\ldots)))) \ldots)))))).) \ldots))) \ldots \ldots \ldots((((\ldots(((\ldots((((\ldots))))))))))$. $>$ p18 minus $(-21.60 \mathrm{Kcal} / \mathrm{mol})$

AUUGGUCUUAAAGGUACCUGAGGUGUGACUGGAAAACCCACCUCCUCCUCCUCUUGUGCUUCUAGCCAGGCACAAGCAG $\ldots(((((\ldots)))).) \cdot((((((\ldots(\ldots(\ldots) \ldots))))))) \ldots \ldots(((((((((((\ldots \ldots))))))))))$. $>$ p19 minus $(-20.90 \mathrm{Kcal} / \mathrm{mol})$

UUAUUGAGGCUUAAGCAGUGGGUUCCCUAGUUAGCCAGAGAGCUCCCAGGCUCAGAUCUGGUCUAACCA $\ldots \ldots \ldots((\ldots)) \ldots((\ldots)) \ldots(((((((((((((((\ldots))))) \ldots))))))))).) \ldots$ $>$ p20 minus $(-21.60 \mathrm{Kcal} / \mathrm{mol})$

GGGAAGUAGCCUUGUGUGUGGUAGAUCCACAGAUCAAGGAUAUCUUGUCUUCUUUGGGAGUGAAUUAGCCCUUCC $((.(((\ldots((((((((((\ldots \ldots)))))))))).) \ldots .)))).) \ldots \ldots((((\ldots(\ldots \ldots)))))$ $>$ p21 minus $(-22.02 \mathrm{Kcal} / \mathrm{mol})$

UCGGGCGCCACUGCUAGAGAUUUUCCACACUGACUAAAAGGGUCUGAGGGAUCUCUAGUUACCAGAGUCACACA

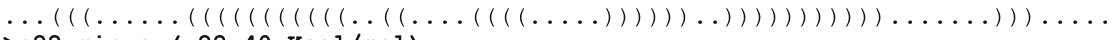
$>$ p22 minus $(-22.40 \mathrm{Kcal} / \mathrm{mol})$

AUGGGAGGGGCAUACAUUGCUUUUCCUACUUUCUGCCACAUGUUUAUAAUUUGUUUUUAUUCUGCAUGGGA .$(((((((((((\ldots \ldots))))))))))) \ldots \ldots \ldots((.(((((\ldots((((\ldots \ldots)))) \ldots)))))))$. $>$ p23 minus $(-22.40 \mathrm{Kcal} / \mathrm{mol})$

UCAACUGGUACUAGCUUGUAGCACCAUCCAAAGGUCAGUGGAUAUCUGACCCCUGGCCCUGGUGUGUAGUU

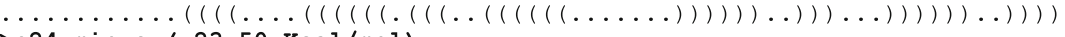
$>$ p24 minus $(-23.50 \mathrm{Kcal} / \mathrm{mol})$

AGGAUUCUUGCCUGGAGCUGCUUGAUGCCCCAGACUGUGAgUUGCAACAGAUGCUGUUGCGCCUCAAUAGC $\ldots \ldots \ldots \ldots((((.((\ldots \ldots \ldots))))).) \cdot\left(\left(\left(\left(\left(\left(\left(.\left(\left(\left(\left(\left(\left(\left(\ldots_{1}\right)\right)\right)\right)\right)\right)\right).\right)\right)\right).\right)\right)\right)\right)$. $>$ p25 minus $(-23.10 \mathrm{Kcal} / \mathrm{mol})$

GGAUUCUUGCCUGGAGCUGCUUGAUGCCCCAGACUGUGAGUUGCAACAGAUGCUGUUGCGCCUCAAUAG $\left.\ldots \ldots \ldots \ldots((((.((\ldots \ldots \ldots))))).) \cdot\left(\left(\left(\left(\left(\left(\left(.\left(\left(\left(\left(\left(\left(\left(\ldots_{1}\right)\right)\right)\right)\right)\right)\right)\right).\right)\right)\right).\right)\right)\right)\right)$ 
Table 8 Comparative analysis of precursor sequence of plus and minus strand

\begin{tabular}{llllllll}
\hline S. no. & Query sequence & Strand type & Aligned with miRbase & Score & $E$ value & Matching alignment length & Match id with miRBase \\
\hline 1. & p7 & Plus & Stem loop sequence & 140 & $1 \mathrm{e}-05$ & $36(34-69)$ & hiv1-mir-H1 \\
2. & $\mathrm{p} 7$ & Plus & Mature miRNA & 68 & 0.92 & $19(35-53)$ & hiv1-miR-H1 \\
3. & $\mathrm{p} 11$ & Plus & Stem loop sequence & 83 & 0.58 & $31(4-34)$ & dps-mir-2544 \\
4. & $\mathrm{p} 11$ & Plus & Mature miRNA & 73 & 0.37 & $20(37-56)$ & cte-miR-2706 \\
5. & $\mathrm{p} 15$ & Minus & Stem loop sequence & 85 & 0.36 & $53(15-67)$ & mmu-mir-715 \\
6. & $\mathrm{p} 15$ & Minus & Mature miRNA & 68 & 0.92 & $19(42-60)$ & oan-miR-1331 \\
7. & $\mathrm{p} 17$ & Minus & Stem loop sequence & 87 & 0.34 & $30(4-33)$ & cel-mir-241 \\
8. & $\mathrm{p} 17$ & Minus & Mature miRNA & 65 & 2.0 & $18(36-53)$ & mdv2-miR-M30 \\
\hline
\end{tabular}

Fig. 6 Predicted miRNA (red colour) in $\mathrm{p} 7$ of plus strand

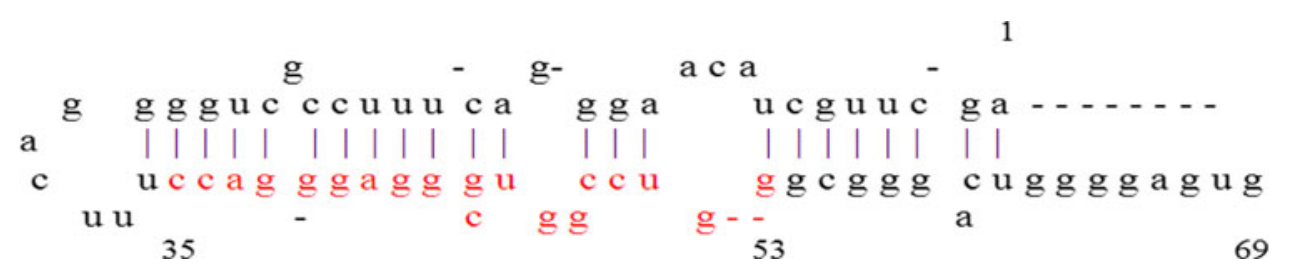

Table 9 Gene target for predicted miRNA at threshold score 19

\begin{tabular}{lllllll}
\hline S. no. & Gene name & Ensemble gene ID & miTG score & Precision & SNR & Gene description \\
\hline 1. & NDUFS7 & ENSG00000115286 & 46.00 & 1.00 & 6.98 & NADH dehydrogenase (ubiquinone) Fe-S protein 7 \\
2. & WNT3A & ENSG00000154342 & 27.00 & 0.95 & 6.98 & Wingless-type MMTV integration site family \\
3. & SUFU & ENSG00000107882 & 20.00 & 0.91 & 6.98 & Suppressor of fused homolog \\
4. & FOXK1 & ENSG00000164916 & 19.00 & 0.91 & 6.98 & Fork head box K1 \\
\hline
\end{tabular}

(19 mer length) is shown in red color (Fig. 6). The predicted miRNA region starts from 35 th to $53 \mathrm{rd}$ position of precursor p7 (plus strand). The sequence of predicted miRNA from complete genome of hiv-1(gil4558520|gblAF033819.3l) is "ccagggaggcguggccugg"

\subsection{Target prediction}

To predict the target of the predicted miRNA, we performed an in silico analysis to detect putative miRNAbinding sites. A higher miTG score corresponds to a higher possibility of being a real target. The precision score ranges from 0 to 1 , and it evaluates the significance of the prediction. The seed match value has been taken at least 7 mer. A signal-to-noise ratio (SNR) and a precision score are calculated for each interaction to provide an estimate of the false-positive rate of each predicted miTG score. A non-strict (default) threshold is set at score 7.3 and a strict threshold at score 19. Predicted miRNAs share 1,510 target sites in 371 genes at threshold score 7.3 but at strict threshold score 19; only 39 targets site in 4 genes have been filtered out. These four target genes are NDUFS7, WNT3A, SUFU, and FOXK1 (Table 9).

NDUFS7 encodes for NADH dehydrogenase, which has important role in mitochondrial activities and programmed cell death (PCD) during somatic embryo development (Chomova and Racay 2010; Petrussa et al. 2009). WNT3A encodes the secreted signaling proteins. These proteins have been caught up in oncogenesis and in several developmental processes, including regulation of cell fate and patterning during embryogenesis (Saitoh et al. 2001). SUFU encodes a component of the sonic hedgehog ( $\mathrm{SHH}$ )/ patched (PTCH) signaling pathway (Delattre et al. 2001). Mutations in genes encoding components of this pathway are lethal for typical development and are related to cancerpredisposing syndromes such as holoprosencephaly, basal cell nevus syndrome, and Greig cephalopolysyndactyly syndrome (Merchant et al. 2004; Taylor et al. 2002, 2004). FOX1 is expressed in immature tissues of brain, eye, heart, lung, and thymus. In adults, FOX1 was mainly expressed in various malignant tissues, such as in the tumors of brain, colon, and lymph node (Huang and Lee 2004).The targets 
of predicted miRNA are associated with the normal development of the organism where mutation in these genes can lead to apoptosis, growth arrest, and cancer predisposing syndrome. This indicates that the predicted miRNA plays an important role in HIV.

\section{Conclusions}

In this study we presented an ANN approach for viral miRNA prediction which is based on the knowledge of experimentally identified pre-miRNA and mature miRNAs. Several filtration steps are taken such as secondary structure feature selection, energy threshold value, etc. The accuracy of validation data set can be enhanced by taking larger dataset as well incorporating more features related to miRNA. Prediction of miRNA is currently a major research area. However, the accurate miRNA targets prediction are more important than predicting the miRNA itself because a single miRNA can target many mRNAs. Therefore, miRNA predictions should genuinely be followed by their target identification. This suggests that mature miRNAs are more conserved in sequence than the pre-miRNAs. This study can help us in understanding the host-pathogen interactions in the virus-infected organisms.

However, there are still restrictions in applying in silico approaches for accurate prediction of miRNAs due to lack of significant and deterministic features of miRNAs and scarcity of biologically relevant dataset. The accuracy of ANN approach for miRNA prediction can be further enhanced by taking into account more consistent features and applicable dataset.

\section{References}

Altschul SF, Gish W, Miller W, Myers EW, Lipman DJ (1990) Basic local alignment search tool. J Mol Biol 215(3):403-410

Ambros V, Bartel B, Bartel DP, Burge CB, Carrington JC, Chen X, Dreyfuss G, Eddy SR, Griffiths-Jones S, Marshall M, Matzke M, Ruvkun G, Tuschl T (2003) A uniform system for microRNA annotation. RNA 9(3):277-279

Anderson D, McNeill G (1995) Artificial neural networks technology. Kaman Sciences Corporation, US

Arendt CW, Littman DR (2001) HIV: master of the host cell. Genome Biol 2:1030.1-1030.4

Bartel DP (2004) MicroRNAs: genomics, biogenesis, mechanism, and function. Cell 116:281-297

Chomova M, Racay P (2010) Mitochondrial complex I in the network of known and unknown facts. Gen Physiol Biophys 29(1):3-11

Delattre M, Briand S, Paces-Fessy M, Blanchet-Tournier MF (2001) The Suppressor of fused gene, involved in Hedgehog signal transduction in Drosophila, is conserved in mammals. Dev Genes Evol 209(5):294-300

Emerman M, Malim MH (1998) HIV-1 regulatory/accessory genes: keys to unraveling viral and host cell biology. Science 280:1880-1884
Griffiths-Jones S (2004) The microRNA Registry. NAR 32:D109D111 (Database Issue)

Griffiths-Jones S, Grocock RJ, van Dongen S, Bateman A, Enright AJ (2006) miRBase: microRNA sequences, targets and gene nomenclature. Nucleic Acids Res 34:D140-D144

Griffiths-Jones S, Saini HK, van Dongen S, Enright AJ (2008) miRBase: tools for microRNA genomics. NAR 36:D154-D158 (Database Issue)

Grishok A, Pasquinelli AE, Conte D, Li N, Parrish S, Ha I, Baillie DL, Fire A, Ruvkun G, Mello CC (2001) Genes and mechanisms related to RNA interference regulate expression of the small temporal RNAs that control C. elegans developmental timing. Cell 106:23-34

Gruber AR, Lorenz R, Bernhart SH, Neuböck R, Hofacker IL (2008) The Vienna RNA Websuite. Nucleic Acids Res 36(suppl 2): W70-W74

Hammond SM, Bernstein E, Beach D, Hannon GJ (2000) An RNAdirected nuclease mediates post-transcriptional gene silencing in Drosophila cells. Nature 404:293-296

He L, Hannon GJ (2004) MicroRNAs: small RNAs with a big role in gene regulation. Nat Rev Genet 5(7):522-531

Huang JT, Lee V (2004) Identification and characterization of a novel human FOXK1 gene in silico. Int J Oncol 25(3):751-757

Hutvagner G, McLachlan J, Pasquinelli AE, Balint E, Tuschl T, Zamore PD (2001) A cellular function for the RNA-interference enzyme Dicer in the maturation of the let-7 small temporal RNA. Science 293:834-838

Ketting RF, Fischer SE, Bernstein E, Sijen T, Hannon GJ, Plasterk RH (2001) Dicer functions in RNA interference and in synthesis of small RNA involved in developmental timing in C. elegans. Genes Dev 15:2654-2659

Khan J, Wei JS, Ringnér M, Saal LH, Ladanyi M, Westermann F, Berthold F, Schwab M, Antonescu CR, Peterson C, Meltzer PS (2001) Classification and diagnostic prediction of cancers using gene expression profiling and artificial neural networks. Nat Med 7(6):673-679

Kiriakidou M, Nelson PT, Kouranov A, Fitziev P, Bouyioukos C, Mourelatos Z, Hatzigeorgiou A (2004) A combined computational-experimental approach predicts human microRNA targets. Genes Dev 18:1165-1178

Kozomara A, Griffiths-Jones S (2011) miRBase: integrating microRNA annotation and deep-sequencing data. NAR 39:D152-D157 (Database Issue)

Lai EC, Tomancak P, Williams RW, Rubin GM (2003) Computational identification of Drosophila microRNA genes. Genome Biol 4(7):R42

Lee Y, Jeon K, Lee JT, Kim S, Kim VN (2002) MicroRNA maturation: stepwise processing and subcellular localization. EMBO J 21:4663-4670

Lee Y, Ahn C, Han J, Choi H, Kim J, Yim J, Lee J, Provost P, Rådmark O, Kim S, Kim VN (2003) The nuclear RNase III Drosha initiates microRNA processing. Nature 425:415-419

Lim LP, Glasner ME, Yekta S, Burge CB, Bartel DP (2003a) Vertebrate microRNA genes. Science 299:1540

Lim LP, Lau NC, Weinstein EG, Abdelhakim A, Yekta S, Rhoades MW, Burge CB, Bartel DP (2003b) The microRNAs of Caenorhabditis elegans. Genes Dev 17:991

Liou S-W, Huang Y-F (2012) An exon/intron disparity framework based on the nucleotide profile of single sequence. Netw Model Anal Health Inform Bioinforma 1:19-25

Maragkakis M, Alexiou P, Papadopoulos GL, Reczko M, Dalamagas T, Giannopoulos G, Goumas G, Koukis E, Kourtis K, Simossis VA, Sethupathy P, Vergoulis T, Koziris N, Sellis T, Tsanakas P, Hatzigeorgiou AG (2009a) Accurate microRNA target prediction correlates with protein repression levels. BMC Bioinform 10:295 
Maragkakis M, Reczko M, Simossis VA, Alexiou P, Papadopoulos GL, Dalamagas T, Giannopoulos G, Goumas G, Koukis E, Kourtis K, Vergoulis T, Koziris N, Sellis T, Tsanakas P, Hatzigeorgiou AG (2009b) DIANA-microT web server: elucidating microRNA functions through target prediction. Nucleic Acids Res 37:W273-W276 (Web Server)

Mendes ND, Freitas AT, Sagot M-F (2009) Current tools for the identification of miRNA genes and their targets. Nucleic Acids Res 37(8):2419-2433

Merchant M, Vajdos FF, Ultsch M, Maun HR, Wendt U, Cannon J, Desmarais W, Lazarus RA, de Vos AM, de Sauvage FJ (2004) Suppressor of fused regulates Gli activity through a dual binding mechanism. Mol Cell Biol 24(19):8627-8641

Nathans R, Chu CY, Serquina AK, Lu CC, Cao H, Rana TM (2009) Cellular MicroRNA and P bodies modulate host-HIV-1 interactions. Mol Cell 34:696-709

Petrussa E, Bertolini A, Casolo V, Krajnáková J, Macrì F, Vianello A (2009) Mitochondrial bioenergetics linked to the manifestation of programmed cell death during somatic embryogenesis of Abies alba. Planta 231(1):93-107

Pfeffer S, Sewer A, Lagos-Quintana M, Sheridan R, Sander C, Grässer FA, van Dyk LF, Ho CK, Shuman S, Chien M, Russo JJ, Ju J, Randall G, Lindenbach BD, Rice CM, Simon V, Ho DD, Zavolan M, Tuschl T (2005) Identification of microRNAs of the herpesvirus family. Nat Methods 2(4):269-276
Saitoh T, Hirai M, Katoh M (2001) Molecular cloning and characterization of WNT3A and WNT14 clustered in human chromosome 1q42 region. Biochem Biophys Res Commun 284(5):1168-1175

Taylor MD, Liu L, Raffel C, Hui CC, Mainprize TG, Zhang X, Agatep R, Chiappa S, Gao L, Lowrance A et al (2002) Mutations in SUFU predispose to medulloblastoma. Nat Genet 31:306-310

Taylor MD, Zhang X, Liu L, Hui CC, Mainprize TG, Scherer SW et al (2004) Failure of a medulloblastoma-derived mutant of SUFU to suppress WNT signaling. Oncogene 23:4577-4583

Thomassen GO, Røsok O, Rognes T (2006) Computational prediction of MicroRNAs encoded in viral and other genomes. J Biomed Biotechnol 4:95270

Van Hulse J, Khoshgoftaar TM, Al Napolitano, Wald R (2012) Threshold-based feature selection techniques for high-dimensional bioinformatics data. Netw Model Anal Health Inf Bioinform 1(1-2):47-61

Wang XW, Zhang J, Li F, Gu G, He T, Zhang XG, Li YD (2005) MicroRNA identification based on sequence and structure alignment. Bioinform 21(18):3610-3614

Zillner S, Sonntag D (2012) Image metadata reasoning for improved clinical decision support. Netw Model Anal Health Inform Bioinform 1:37-46 